\title{
From the perspective of embryonic tendon development: various cells applied to tendon tissue engineering
}

\author{
Fangjie Qi ${ }^{1 \#}$, Zhantao Deng ${ }^{1 \#}$, Yuanchen $\mathrm{Ma}^{1}$, Shuai Wang ${ }^{1}$, Chang Liu ${ }^{1}$, Fengjuan Lyu ${ }^{1}$, \\ Tao Wang ${ }^{1,2}$, Qiujian Zheng ${ }^{1,2}$ \\ ${ }^{1}$ Department of Orthopedics, Guangdong Provincial People's Hospital, Guangdong Academy of Medical Sciences, School of Medicine, South China \\ University of Technology, Guangzhou Higher Education Mega Centre, Guangzhou 510006, China; ${ }^{2}$ Centre for Orthopaedic Translational Research, \\ School of Biomedical Sciences, University of Western Australia, Nedlands, Western Australia, Australia \\ Contributions: (I) Conception and design: F Qi, T Wang, Q Zheng; (II) Administrative support: None; (III) Provision of study materials or patients: \\ Z Deng; (IV) Collection and assembly of data: F Qi; (V) Data analysis and interpretation: F Qi; (VI) Manuscript writing: All authors; (VII) Final \\ approval of manuscript: All authors. \\ \#These authors contributed equally to this work. \\ Correspondence to: Qiujian Zheng or Tao Wang. Guangdong Provincial Hospital, Guangdong Academy of Medical Sciences, School of Medicine, \\ South China University of Technology, 106 Zhongshan 2nd Rd., Yuexiu Qu, Guangzhou 510080, China. Email: zhengqiujian@gdph.org.cn; \\ tao.wang@uwa.edu.au.
}

\begin{abstract}
There is a high risk of injury from damage to the force-bearing tissue of the tendon. Due to its poor self-healing ability, clinical interventions for tendon injuries are limited and yield unsatisfying results. Tissue engineering might supply an alternative to this obstacle. As one of the key elements of tissue engineering, various cell sources have been used for tendon engineering, but there is no consensue concerning a single optimal source. In this review, we summarized the development of tendon tissue from the embryonic stage and categorized the used cell sources in tendon engineering. By comparing various cell sources as the candidates for tendon regeneration, each cell type was found to have its advantages and limitations; therefore, it is difficult to define the best cell source for tendon engineering. The microenvironment cells located is also crucial for cell growth and differentiation; so, the optimal cells are unlikely to be the same for each patient. In the future, the clinical application of tendon engineering might be more precise and customized in contrast to the current use of a standardized/generic one-size-fits-all procedure. The best cell source for tendon engineering will require a case-based assessment.
\end{abstract}

Keywords: Tendon; tissue engineering; stem cells; cell types

Submitted Oct 28, 2019. Accepted for publication Dec 06, 2019.

doi: $10.21037 /$ atm.2019.12.78

View this article at: http://dx.doi.org/10.21037/atm.2019.12.78

\section{Introduction}

Tendons are unique connective tissues that not only connect the muscles and bones, but they are also important for maintaining posture and locomotion through transmitting muscle-contraction force to the skeleton. The tendon is composed predominantly of tenocytes surrounded by extracellular matrices (ECM) such as collagen I fibers, proteoglycans, and glycoproteins. It forms a solid structure and can support high repetitive mechanical loading (1).
However, tendon tissue has poor healing ability and limited regenerative ability; the damage may become irreversible, and the healing process will be difficult, leading to chronic disability. Although the current wide use of topical or systemic anti-inflammatory drugs can reduce the perception of pain, and surgical repair of ruptured tendons through autograft or allograft seems to maintain the physiological function of the tendon (2), the functional, structural, and biochemical properties of the damaged tendon cannot be fully restored to an uninjured status (3). In addition, the 
repair process may induce the formation of scar tissue (4), of which the tensile strength is only one-third that of an undamaged tendon, which can be a triggering factor for a large number of cases of secondary damage (5). Furthermore, autograft may cause donor site morbidity, while allograft may elicit an immune rejection (6).

Therefore, tissue engineering has been explored in an attempt to improve tendon healing and eventually reach a complete biological repair. A classic tissue engineering (7) technique is to culture seed cells in an engineered structure that is made of a biodegradable scaffold with the supplementation of growth factors or either mechanical or chemical factors to promote cell proliferation and differentiation in vitro before it is transplanted into the damaged site in vivo. Subsequently, the scaffold could be substituted by a newly formed organization that can replace damaged tissues and ultimately reconstitute the tissue functions (8). In tendon tissue engineering, cells also play a vital role in producing ECM for the reconstruction of the tendon tissue structure (9). Thus, the source of the seed cells becomes one of the key points in tendon tissue engineering. To find more potential candidates suitable for tendon tissue engineering, we explore the embryological origin of the limb tendons, analyze the developing processes of tendons, and summarize the availability of various types of seed cells using tendon tissue engineering. So, at the beginning of the article, we will reconstruct the development of the tendon from the embryo to maturity and explain the availability of these cells.

\section{Embryological origin and development of tendons}

Human embryonic development begins with the cleavage of the fertilized egg. It then divides into many smaller cells leading to the formation of the blastula. Through the cell movements of gastrulation, the embryo is rearranged to form the three main germ layers: the ectoderm, mesoderm, and endoderm. The mesoderm then gives rise to chordamesoderm, paraxial mesoderm, intermediate mesoderm, and lateral plate mesoderm. With the establishment of body axes and formation of the neural tube, limb buds arise from lateral plate mesoderm at the appropriate levels along the ventral domain of the body (10). The early limb bud has two major components: an outer layer of ectodermal epithelial cells, and a core of loose undifferentiated mesenchymal cells derived from the lateral plate mesoderm $(11,12)$. The skeletal elements and connective tissues (including tendon structure) of the limb develop from these mesenchyme cells, but the limb muscles are derived from the somites which stem from the region of paraxial mesoderm and migrate into the limb bud. Therefore, the origin of tendon tissue is the same as that of bones, cartilage, and any other connective tissues, but not muscle. Analysis of the expression of scleraxis (Scx), a tendon specific transcription factor, revealed that the limb tendon progenitors originate from the subectodermal mesenchyme in proximomedial domains of the limb bud (13). Regulated by multiple morphogens and growth factors, three pairs of tendon primordium appear in a proximo-distal sequence in the developing limb $(14,15)$. Proximal and intermediate tendon primordium appears the earliest on the dorsal side around limb junction. Following closely, intermediate tendon primordium appears on the ventral side around intermediate limb junction. The ventral proximal tendon primordium forms after the appearance of ventral intermediate tendon primordium. Subsequently, distal tendon primordium appears at both the dorsal and ventral sides of the distal limb. After the stimulation of growth factors and mechanical stress (16), the three pairs of tendon primordium will then differentiate into adult tendon structure in a proximal-to-distal sequence in the developing limb (Figure 1).

\section{Various types of seed cells for tendon tissue engineering}

Based on the process of limb tendon development, tenocytes originate from mesenchymal stromal cells (MSCs), which are also progenitor cells of skeletal elements and other connective tissues. Limb bud MSCs are derived from the lateral plate mesoderm of the embryo. These cells at different stages of tendon differentiation are candidate types of seed cells in tendon tissue engineering.

In the process of tendon development, tenocytes are the terminal product and the major component of the tendon's structure. Dermal fibroblasts belong to the class of mesenchymal cells, which are differentiated from MSCs. As dermal fibroblasts are labile cells and have the same precursors as tenocytes, they can be used in tendon repair (17).

Tenocytes are derived from tendon progenitor cells, which are called tendon-derived stem cells (TDSCs) (18). TDSCs are a type of stem cell that possess some characteristics of multipotent stem cells (19). Defined by the specific stem-cell niche, MSCs can be classified into bone marrow-derived mesenchymal stromal cells (BMSCs), adipose-derived stem cells (ADSCs), TDSCs, and other 


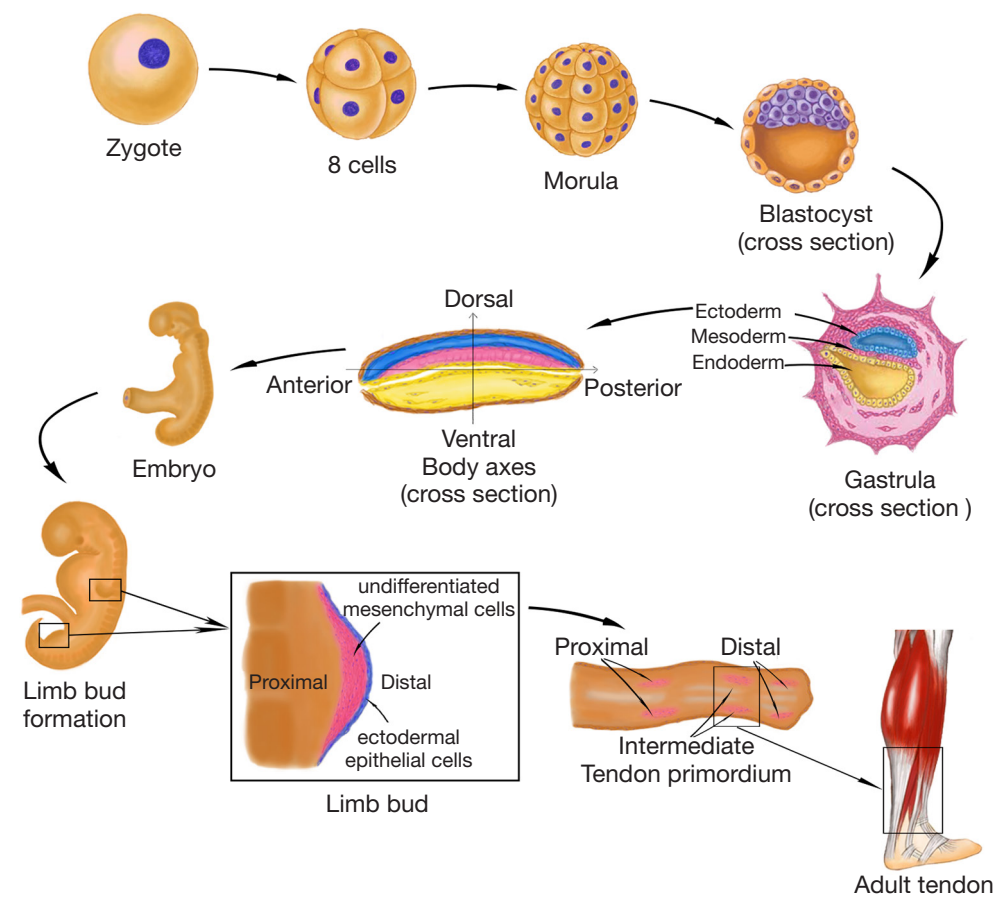

Figure 1 The process of human limb tendon development.

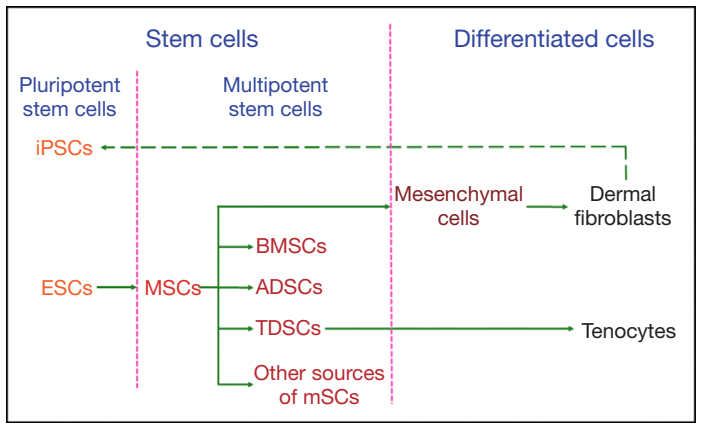

Figure 2 The derivation of tenocytes from ESCs and classification of cell types. Green solid arrows show the direction of differentiation, green dotted arrow shows that iPSCs can be obtained from dermal fibroblasts by dedifferentiation. The deepening of the font color means that the ability of cells to proliferare and differentiate is gradually reduced. ESCs, embryonic stem cells; iPSCs, induced pluripotent stem cells; MSCs, mesenchymal stromal cells; BMSCs, bone marrow-derived mesenchymal stromal cells; ADSCs, adipose-derived stem cells; TDSCs, tendon-derived stem cells.

sources of MSCs (20). These MSC-derived stem cells can be differentiated into tenocytes under appropriate stimulation in vitro.
Another available cell type is pluripotent stem cells, which can differentiate into all types of cells constituting the human body. These include embryonic stem cells (ESCs) and induced pluripotent stem cells (iPSCs), which can be derived from differentiated human dermal fibroblasts (21).

In summary, the available seed cells for tendon tissue engineering include ESCs, iPSCs, MSCs (included BMSCs, ADSCs, and TDSCs), dermal fibroblasts, and tenocytes, as summarized in Figure 2. Next, we will discuss the respective advantages and disadvantages of these different cell types.

\section{Comparison between different cell types}

The cells in the human body can be divided into two categories based on their differentiation status (Table 1). One is differentiated cells, such as tenocytes and dermal fibroblasts, which lack self-renewal capability in vivo. Differentiated tenocytes and dermal fibroblasts can be expanded in vitro in the presence of serum but have a limited expansion capacity. Culturing in vitro with the supplementation of growth factors may activate their ability of proliferation, but these cells still lack the capacity of differentiating into other cell types. Besides, their phenotype may change, which will cause a deficiency in their functions with increasing passaging (18). The 
Table 1 Relative comparison between two human cell types using tissue engineerin

\begin{tabular}{llll}
\hline Cell types & Category & Advantage & Disadvantage \\
\hline $\begin{array}{lll}\text { Differentiated } \\
\text { cells }\end{array}$ & $\begin{array}{l}\text { Tenocytes, dermal } \\
\text { fibroblasts }\end{array}$ & $\begin{array}{l}\text { Low risk of teratoma } \\
\text { and ectopic tissue formation }\end{array}$ & $\begin{array}{l}\text { Terminal differentiated cells } \\
\text { Limited proliferation rate }\end{array}$ \\
& & & Phenotype change and functional loss during expansion in vitro \\
Stem cells & ESCs, MSCs & Proliferative capacity & Teratoma and ectopic tissue formation \\
& & Multidirectional differentiation potential & \\
\hline
\end{tabular}

ESCs, embryonic stem cells; MSCs, mesenchymal stromal cells.

other is stem cells, which can replicate themselves as well as differentiate into specialized cells under appropriate conditions (22). At the same time, their ability to proliferate and differentiate is difficult to control in vivo, which makes them susceptible to forming of tumor and undesired tissue cells during differentiation.

\section{Differentiated cells}

\section{Tenocytes}

Tenocytes are highly specialized mesenchyme-derived cells with important roles in synthesizing ECM and maintaining tendon structures in vivo (23). Cao et al. constructed tissueengineered artificial tendons for the first time (24), but they also indicated that tenocytes are relatively difficult to grow and expand in vitro. Furthermore, some researchers indicated that with increased passaging, the gene expression of tendon-associated proteins such as collagen type I (col I), collagen type III (col III), tenascin, and tenomodulin (TNMD) exhibited a trend of decrease (25). In addition, there is also a change in the tenocyte cell phenotype, represented by more rounded cell morphologies instead of longer and thicker configurations (18). Thus, many researchers have explored the strategies to promote proliferation and at the same time, support a stable phenotype of tenocytes. At present, it has been reported that thyroid hormone T3, transforming growth factor (TGF)-b1, glycine, platelet-rich plasma, and insulinlike growth factor 1 could enhance the proliferation and differentiation capacity of tenocytes, as well as stimulate the secretion of ECM (23,26-29). Mechanical force can also regulate tenocyte differentiation (30).

\section{Dermal fibroblasts}

Dermal fibroblasts are terminally differentiated cells that originate from mesoderm. In vitro, dermal fibroblasts exhibit similar cell morphology as tenocytes and mainly produce col I and col III. Compared with tenocytes, the harvest of dermal fibroblasts is less defective as only an easily accessible, small piece of skin is needed. Also, the cell expansion is less likely during in vitro culture (31). It has been revealed that there is no difference in their gross view between neo-tendon tissues engineered by human dermal fibroblast or tenocytes. There was also no difference found in the histologic structure, collagen superstructure, or mechanical property under the static strain in vitro (32-34). Therefore, researchers have used dermal fibroblastengineered tendon to repair animal tendon defect, and the results are satisfactory in that the tensile stiffness and maximum load are expressly higher than those of nondermal fibroblast scaffolds (35-38).

When dermal fibroblasts and tenocytes are compared, both originate from mesoderm and have similar morphologies (36), and it was determined that dermal fibroblasts were more advantageous compared to tenocytes. First, dermal fibroblasts have good proliferative capacity and self-renewal potential (39). Second, dermal fibroblasts have been shown to be easy to harvest with no major tissue defects at the donor site since the skin can regenerate in a short time (40). In contrast, tenocytes are more difficult to collect because the density of tenocytes in a tendon is low, and there is an issue of donor site morbidity (41). However, dermal fibroblasts have a disadvantage in that they may produce fibrotic ECM which is involved in scar formation (42) (Table 2).

\section{Stem cells}

According to the differentiation potential, stem cells can be divided into distinct kinds of cell types. Pluripotent stem cells can differentiate into all cell types of all organs, 
Table 2 Comparison between two differentiated cells types

\begin{tabular}{lll}
\hline Cell types & Advantage & Disadvantage \\
\hline Tenocytes & Major cell type in the tendon & Low cell number in the tendon \\
& Produce ECM for tendon function maintenance & Donor site morbidity \\
Dermal fibroblasts & Easy to harvest from skin punch biopsy & Not tenocyte \\
& Production similar to the tenocyte & Unstabled phenotype \\
& & Scar formation \\
\hline
\end{tabular}

ECM, extracellular matrix.

Table 3 Comparison between stem cells

\begin{tabular}{|c|c|c|c|}
\hline Cell types & Category & Advantages & Disadvantages \\
\hline iPSCs & - & $\begin{array}{l}\text { Unlimited proliferative ability } \\
\text { Pluripotent } \\
\text { No ethical concern for tissue source }\end{array}$ & $\begin{array}{l}\text { Risk of teratoma formation } \\
\text { Genotype change in the transfection process } \\
\text { Low efficiency of the transfection process } \\
\text { Less reported in tendon regeneration }\end{array}$ \\
\hline MSCs & $\begin{array}{l}\text { BMSCs } \\
\text { ADSCs } \\
\text { TDSCs }\end{array}$ & $\begin{array}{l}\text { Low cancerization rate } \\
\text { Easily available tissue source for cell isolation } \\
\text { No ethical concern for tissue source }\end{array}$ & $\begin{array}{l}\text { Senescence and loss of stem cell characteristics as the } \\
\text { number of passages increase in vitro }\end{array}$ \\
\hline
\end{tabular}

ESCs, embryonic stem cells; iPSCs, induced pluripotent stem cells; MSCs, mesenchymal stromal cells; BMSCs, bone marrow-derived mesenchymal stromal cells; ADSCs, adipose-derived stem cells; TDSCs, tendon-derived stem cells.

like ESCs and iPSCs (43). Multipotent stem cells can develop into many cell types within a particular lineage. For instance, the MSCs can give rise to bone cells, cartilage cells, adipose cells, and tendon cells. As ESCs have unlimited self-renewal potential and can differentiate into cells of all three embryonic germ layers (44), the risk of developing teratoma is expected to increase (45).

Meanwhile, considering that ESCs originate from the blastocyst, the harvest of human ESCs requires the destruction of the embryos, which may give rise to ethical concerns (46). iPSCs derived from differentiated cells may obviate these ethical issues. But, as they are pluripotent stem cells, the problem of forming teratoma during proliferation and differentiation remains unsolved. Furthermore, there are serious problems in the process of inducing iPSC formation (47).

By contrast, MSCs are more safe due to their limited proliferation capacity and the restricted multilineage differentiation potential of cells of the mesodermal lineage $(48,49)$. The use of MSCs also bypasses certain ethical obstacles as they are adult stem cells that can be harvested from non-embryonic sources (50). However, it has been accepted that MSC expansion in culture results in their accelerated aging, which will cause the deficiency of proliferation and multilineage differentiation potential $(51,52)$ (Table 3).

\section{ESCs}

ESCs can give rise to all tissues derived from the three germ layers (53). Because of this, ESCs hold great promise as seed cells for tendon tissue engineering (54). Thus, how to differentiate them into the tendon lineage has become a key point. Some researchers have investigated a stepwise differentiation approach by first inducing human ESCs to differentiate into MSCs, and subsequently allowing the MSCs to form tendon-like tissues. The results in vivo and in vitro showed that human ESC-derived MSCs exhibited tenocyte-like morphology and positively expressed tendon-related gene markers such as Scx, col I and col III, as well as other mechanosensory structures and molecules $(55,56)$. Moreover, the formation of teratomas could be avoided if ESCs are induced into MSCs before the transplantation (55). 
In addition, they demonstrated that the use of dynamic mechanical stress (1 HZ, $10 \%$ for $2 \mathrm{~h}$ /day) and bone morphogenetic protein (BMP)12 and BMP13 could promote differentiation of human ESCs into tenocytes (57-60).

\section{iPSCs}

The use of ESCs may be limited due to the need to sacrifice an embryo, which has aroused some ethical controversy. The discovery of iPSCs resolves the ethical problem of using ESCs, and recently, researchers were able to generate iPSCs from terminally differentiated cells $(21,61)$. However, as their iPSCs were generated using retroviruses or lentiviruses (62), it might cause mutagenesis that would pose a risk for adverse effects in therapy (63). The efficiency of the transfection process also remains low. Thus, for the purpose of the safety of cell transplantation therapy, mRNA-delivered transcription factors have been applied to generate integration-free iPSCs $(64,65)$. While these studies address some of the issues raised by the use of iPSCs in regenerative medicine, it has not been reported in tendon tissue engineering. For now, iPSCs are being used as a potential seed cell source for tendon regeneration research.

\section{MSCs}

MSCs are non-hematopoietic adult stem cells derived from the mesoderm germinal layer that can differentiate into mesenchymal-derived cell types and have the ability to selfrenew (66). The membrane surface of MSCs expresses several antibodies, such as stromal cell antigen-1, CD271, stage-specific embryonic antigen-4, CD146, and so on, which can be considered as specific markers of MSCs $(67,68)$. MSCs were initially isolated from bone marrow as precursors of stromal elements (69). From recent research, it is now clear that MSCs can be isolated from a wide range of adult and perinatal mesenchymal tissues, including those of bone, synovial membranes, periosteum, adipose tissue, tendons, skeletal muscles, and others (70). The use of MSCs for tendon repair has been extensively explored and may promote tendon regeneration.

\section{BMSCs}

The BMSCs have active self-proliferative and multidifferentiate capacity. The use of autologous BMSCs in the animal model could induce matrix production and organization of injured tendon as well as restore histological structure and function (71). Because of the easy isolation from bone marrow and the enrichment of colony-forming units, BMSCs have become an attractive cell source for tissue engineering approaches. However, BMSCs may differentiate into osteoblasts instead of the expected tenocyte lineage when transplanted into the injured tendon, and form ectopic bone in vivo (72,73). To overcome such issue, a number of approaches are presently being developed to navigate the tenogenic differentiation of BMSCs (74). They have shown that cyclical uniaxial stretching and BMP14, TGF- $\beta$, connective tissue growth factor (CTGF), vascular endothelial growth factor (VEGF), and myostatin can initiate and maintain highly efficient growth and differentiation of BMSCs towards tenocytes in vitro (75-80). Furthermore, Zhang et al. (78) and Xie et al. (81) used a 3D culture environment combined with BMSCs sheets that have therapeutic effects on improving the healing quality of the tendon in vivo.

\section{ADSCs}

ADSCs are advantageous in tissue engineering due to their multipotency, high proliferation, easily isolated amounts of cells from the subcutaneous tissue, and low donor site morbidity $(82,83)$. ADSCs have been regarded as one type of seed cell for tendon tissue engineering that can differentiate into tenocytes in vivo and increase the tensile strength of the repaired tendon $(84,85)$. However, their innate tendency towards adipogenesis may hinder the application of ADSCs in tendon regeneration $(1,86)$. For this purpose, intense research was conducted to overcome this obstacle. Yu et al. confirmed that hypoxia or activating the expression of hypoxia-inducible factor-1 could improve the tenogenesis of ADSCs (87). Additionally, several studies have indicated that the supplementation of growth factors could improve cell proliferation of ADSCs and promote tendon repair efficiency (88-90). Tendon ECM components (91) and TNMD (92) enhance the proliferation and tenogenesis of ADSCs. Physical stimulation such as uniaxial tensile cyclic loading (2\% strain and $0.1 \mathrm{~Hz}$ frequency) (93) and extracorporeal shock waves in tenogenic medium (94) can also boost the differentiation of human ADSCs toward tendon-like cells.

\section{TDSCs}

TDSCs isolated from human tendons have general stem cell characteristics such as clonogenicity, multipotency, and self-renewal capacity $(95,96)$. TDSCs can spontaneously 
Table 4 Comparison between MSCs

\begin{tabular}{lll}
\hline Cell types & Advantage & Disadvantage \\
\hline BMSCs & Extensive research history & Ectopic bone formation in vivo \\
& $\begin{array}{l}\text { A large number of research results } \\
\text { are available for reference }\end{array}$ & $\begin{array}{l}\text { Small quantity of viable cell yield in vitro } \\
\text { Harvested by an invasive method with many complications on donor site }\end{array}$ \\
& High cell yield and excellent expansion ability & Ectopic adipose formation \\
& Harvest through liposuction aspirates with & \\
& less trauma and at lower risk & \\
TDSCs & Lineage superiority & Donor site morbidity \\
& Express higher tendon-related gene markers & Less practical research data \\
& and differentiation factors & \\
Other sources & New source & Lack of research data \\
of MSCs & & \\
\hline
\end{tabular}

BMSCs, bone marrow-derived mesenchymal stromal cells; ADSCs, adipose-derived stem cells; TDSCs, tendon derived stem cells.

regenerate tendon-like tissue structures in vivo and avoid differentiating into other kinds of cell lines. Extensive progress and deep insights have emerged in the study of these cells for tendon tissue engineering and regeneration (19). ECM (97), biglycan (98), hepatocyte growth factor (99), and mechanical stimulation (100) that can promote TDSCs proliferation and tenogenic differentiation in vitro have been verified in research. CTGF and ascorbic acid can enhance the survival time, proliferation, and migration abilities of TDSCs in vivo (101). Recently, Zhang et al. demonstrated that genetic alterations of TDSCs following culture expansion could be prevented by pretreating TDSCs with histone deacetylase inhibitor, to retain their ability to accelerate tendon repair in vivo (102). Furthermore, Yin et al. identify a subpopulation of nestin+ TDSCs which exhibited a superior tenogenic capacity compared with nestin- counterparts (103). This study not only redefines the subpopulation of tendon stem cells but also provides new insights into a novel cell line for tendon tissue engineering.

\section{Other sources of MSCs}

Moshaverinia et al. tested the capacity of encapsulated dental MSCs to differentiate into tendon tissue in vitro and in vivo. Their findings indicate that periodontal ligament and gingival tissue-derived MSCs can be considered as suitable stem cell sources for tendon engineering (104). Chen et al. explored dental pulp stem cells (DPSCs) for potential application in tendon tissue engineering and found that mature tendon-like tissue was formed after transplantation of DPSCs in a special fiber scaffold constructs under mechanical loading in a mouse model (105).

\section{Comparison of the potential of various types of MSCs for tendon tissue engineering}

MSCs can be harvested from various sources, such as bone marrow, adipose fat, and tendon tissue. They have paracrine effects, including immunomodulation, promoting angiogenesis, or suppressing inflammation and immune cell functions through secretory factors (106). BMSCs were the first discovered MSCs and the most widely used seed cells in tissue engineering. They are easy to harvest through bone marrow aspiration, but their limitations include relatively small quantities of viable cell yield through an invasive method, which cause many complications on the donor site and ectopic ossification after transplantation in vivo $(107,108)$. ADSCs can be obtained from liposuction aspirates, which is less invasive and involves lower risk. With the relatively large storage of adipose tissue in vivo, a mass of ADSCs can be isolated and expanded in vitro $(109,110)$. However, the main disadvantage of ADSCs is their tendency to undergo adipogenesis in vivo (111). TDSCs express higher tendon-related markers and differentiation factors than BMSCs and ADSCs $(112,113)$, but the main drawback of TDSCs is that their isolation will lead to the injury of the donor site (114). Overall, there is not enough data from practical research on TDSCs to make firm or extensive conclusions (Table 4).

\section{Conclusions}

Tendons are an important component in the musculoskeletal system. Due to the limited regenerative ability of tendons, the healing process after an injury is slow, and the functions of the tendon are likely to be compromised. This results in 
a serious decline in the quality of life of patients. Cell-based tendon tissue engineering is a promising research area that aims to deliver adequate, regeneration-competent cells to the injured tendon and ultimately promote the restoration of its functions (20). This review provides an overview of tendon tissues from an embryonic stage and discusses the relative merits of each of the candidates that could be used as cell sources for tendon regeneration. Depending on the characteristics of each cell type, the researchers tested the most appropriate mechanical stimulation conditions and selected the different biological factors for improving the healing quality of the damaged tendon and reducing the adverse reactions during use (Table 5). Some major findings are described below: Mechanical stimulation will enhance the tenocyte differentiation in different cell types. Growth factors can promote cell proliferation in differentiated cells. However, in stem cells, growth factors not only enhance tenogenic differentiation but also increase the rate of cell growth. Furthermore, cell ECM also can accelerate cell proliferation and the tenogenesis of MSCs. Therefore, the results of these studies may provide new concepts or methods in clinical treatment to improve the level of tendon repair in patients. Doctors can select different cells to treat tendon disease by measuring a patient's expression level of growth factors, tendon transcription factors, the expression of collagen and cytoskeletal proteins in the extracellular matrix, and the microenvironment of the tendon. Clinical treatment of tendon injury can also provide an appropriate cell plan by avoiding the drawbacks of these seed cells. Massive ectopic ossification and fatty vesicles forming in the tendinopathy indicate that BMSCs and ADSCs are not the best choices in the treatment of tendinopathy (115). In addition, scar formation and disorganization of fibers at tendon damage or stump are factors to be considered when selecting candidate cells.

Since there is such a large variety of individual complexity for each of the patients undergoing clinical treatment, it is difficult to define optimal cell sources in tendon engineering. There are various types of tendon damage, including tendon rupture, tear, degeneration, tendinopathy, etc., which might change the cellular status at the damage site (116), and eventually affect the treatment outcomes. Furthermore, stem cell quality varies widely for different individuals. Therefore, there is no best cell source for tendon engineering in general. The determination of the most suitable cell source should be based on a comprehensive assessment of each patient. In the future, clinical treatment of tendon injury by tissue engineering

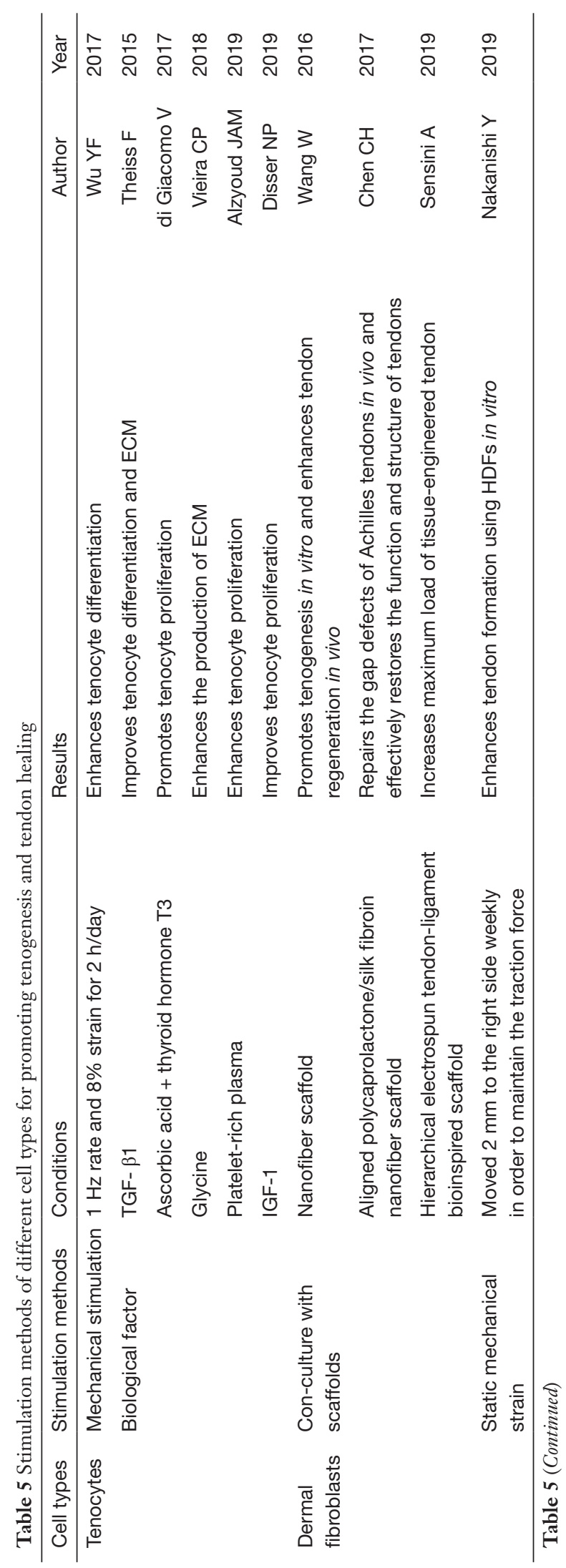




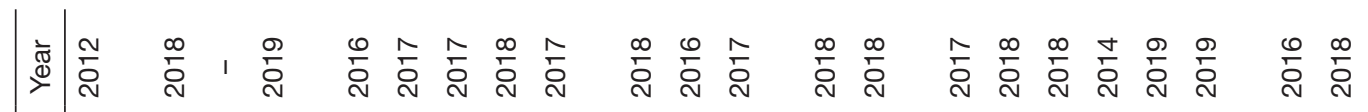

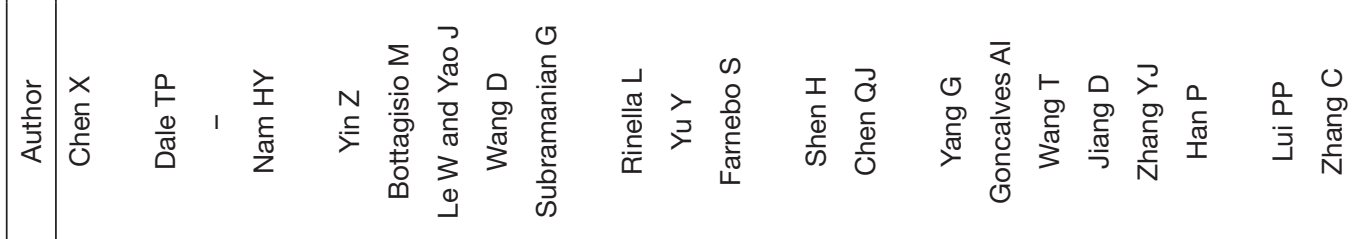

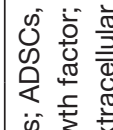

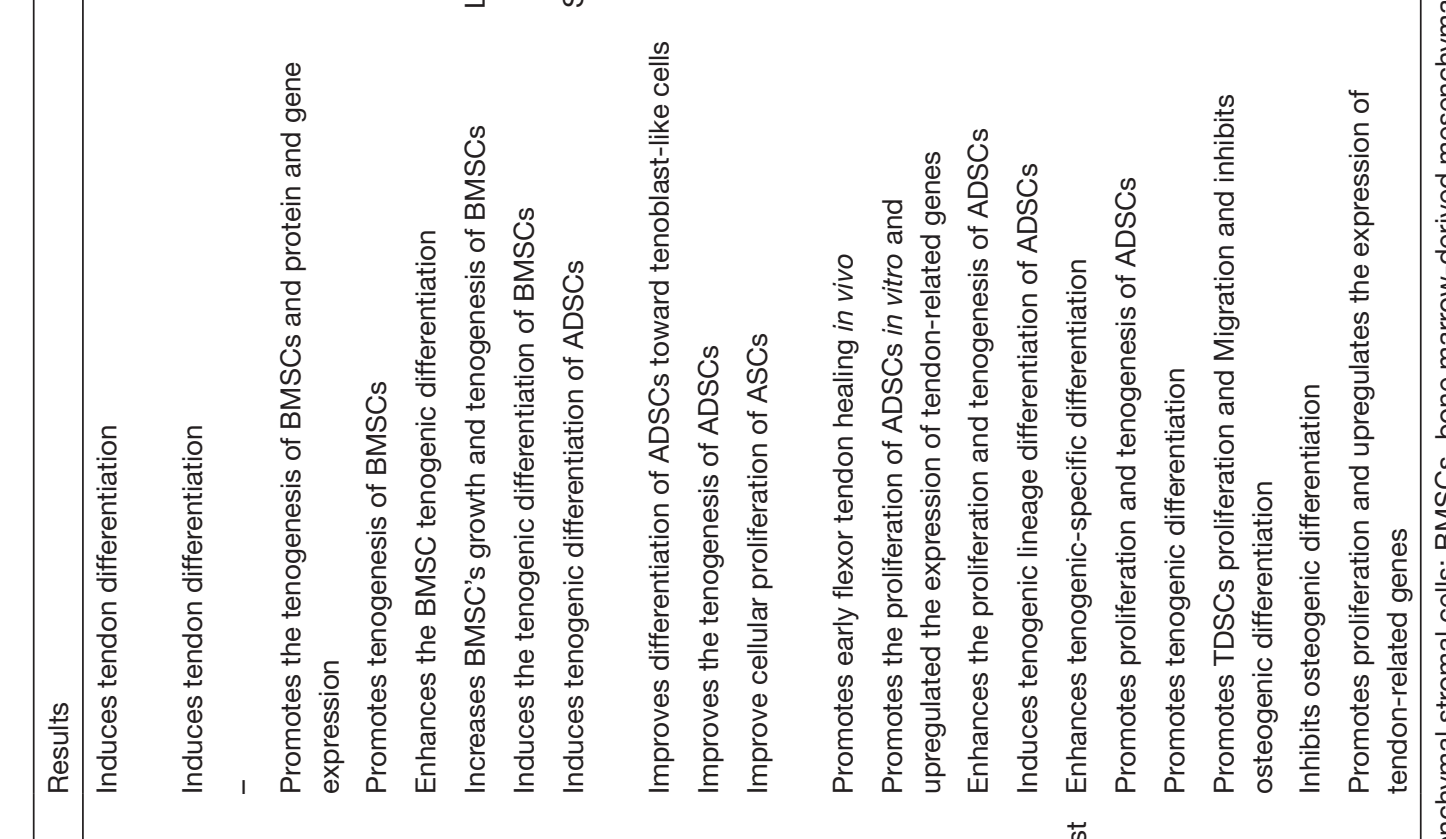
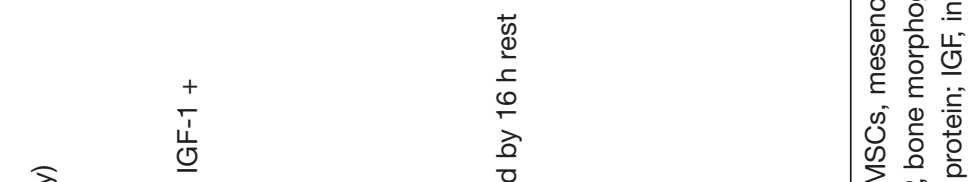

要

is

W 8 을

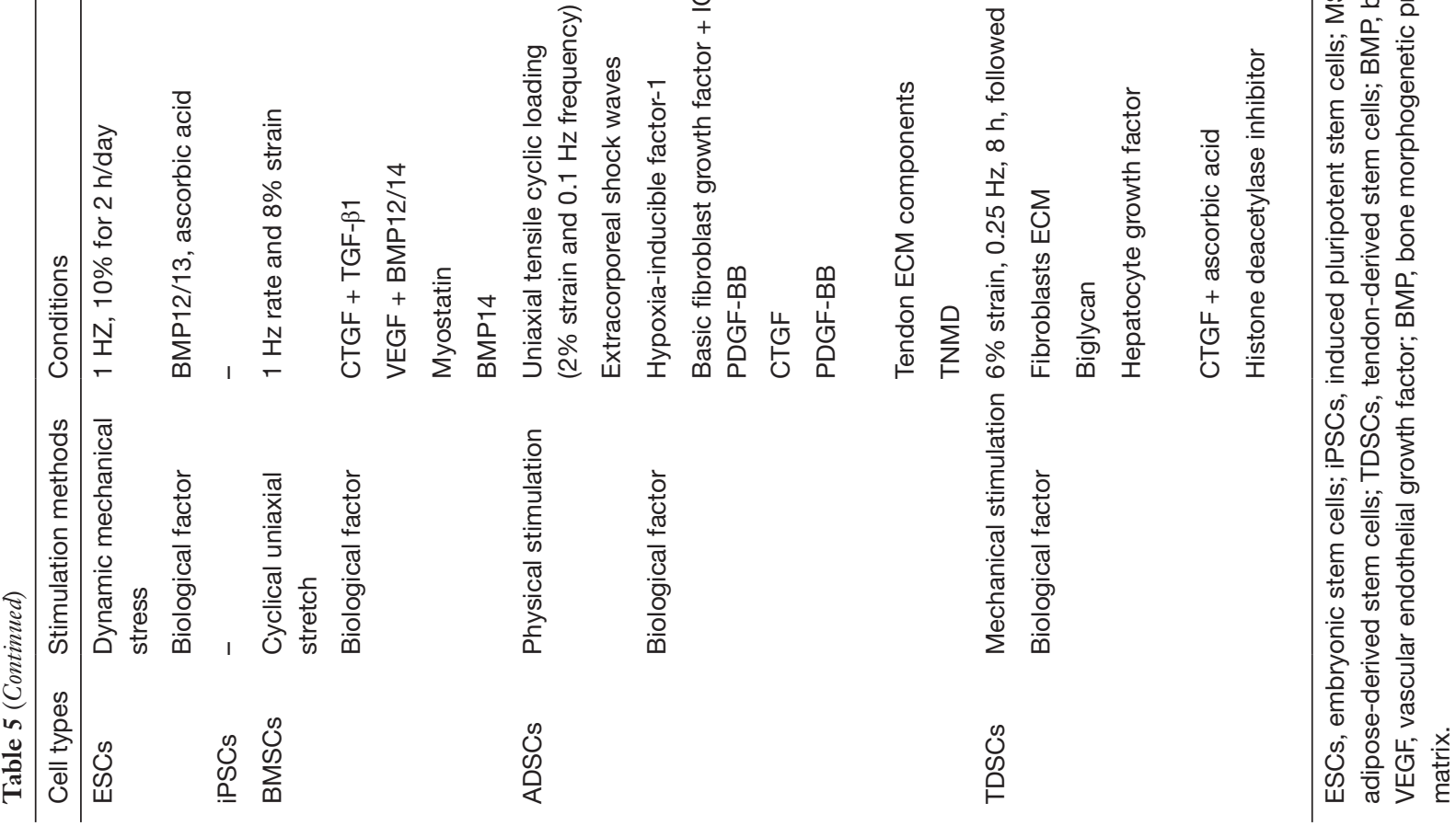

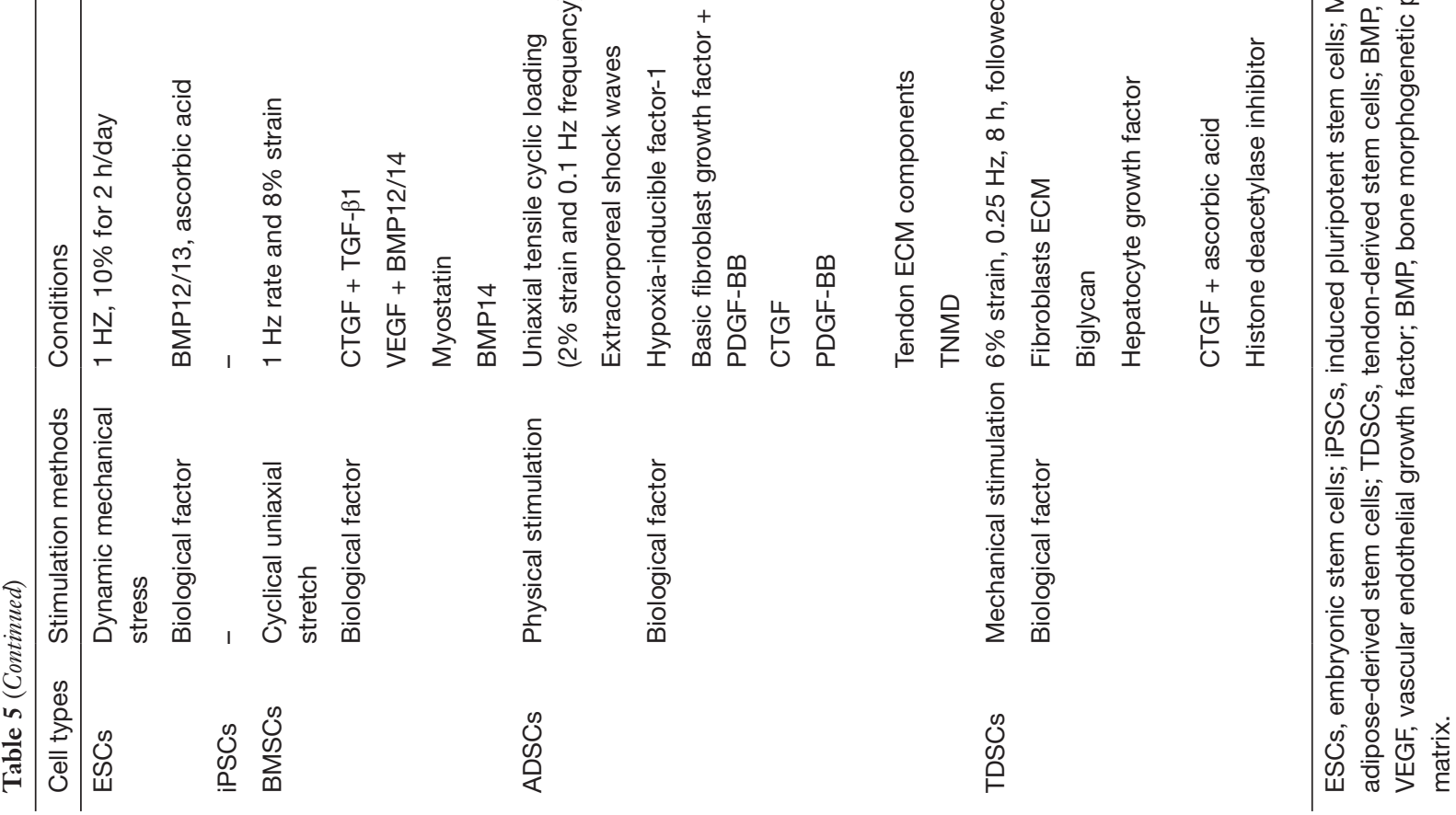


should be customized designed on a case-by-case basis to achieve the best clinical outcomes.

\section{Acknowledgments}

Funding: This study was supported by the National Natural Science Foundation of China (81802214;81702191; $81371991)$, the National Natural Science Foundation of China Youth Science Foundation (81802222), the Major Program of Science and Technology of Guangdong (2015B020225007), the Natural Science Foundation of Guangdong Province (2018A030310694), the Fundamental Research Funds for the Central Universities, South China University of Technology (2018MS70), and the Guangdong Medical Science and Technology Research Foundation (2018114214430383). The Fundation of Traditional Chinese Medicine of Guangdong Province (20191004), The scientific Foundation of Guangdong Provincial People's Hospital (2017bp01), The Outstanding Young Talents Foundation of Guangdong Provincial People's Hospital (KJ012019091), Prrogam of Science and Technology of Guangzhou (201904010424).

\section{Footnote}

Conflicts of Interest: The authors have no conflicts of interest to declare.

Ethical Statement: The authors are accountable for all aspects of the work in ensuring that questions related to the accuracy or integrity of any part of the work are appropriately investigated and resolved.

\section{References}

1. Docheva D, Muller SA, Majewski M, et al. Biologics for tendon repair. Adv Drug Deliv Rev 2015;84:222-39.

2. Nourissat G, Berenbaum F, Duprez D. Tendon injury: from biology to tendon repair. Nat Rev Rheumatol 2015;11:223-33

3. Wei Z, Reisdorf RL, Thoreson AR, et al. Comparison of Autograft and Allograft with Surface Modification for Flexor Tendon Reconstruction: A Canine in Vivo Model. J Bone Joint Surg Am 2018;100:e42.

4. Juneja SC. Cellular distribution and gene expression profile during flexor tendon graft repair: A novel tissue engineering approach $\left({ }^{*}\right)$. J Tissue Eng 2013;4:2041731413492741.
5. Andarawis-Puri N, Flatow EL, Soslowsky LJ. Tendon basic science: Development, repair, regeneration, and healing. J Orthop Res 2015;33:780-4.

6. Chang SK, Egami DK, Shaieb MD, et al. Anterior cruciate ligament reconstruction: allograft versus autograft. Arthroscopy 2003;19:453-62.

7. Langer R, Vacanti JP. Tissue engineering. Science 1993;260:920-6.

8. Glass ZA, Schiele NR, Kuo CK. Informing tendon tissue engineering with embryonic development. J Biomech 2014;47:1964-8.

9. Sharma P, Maffulli N. Biology of tendon injury: healing, modeling and remodeling. J Musculoskelet Neuronal Interact 2006;6:181-90.

10. Hwang SH, White KA, Somatilaka BN, et al. The G protein-coupled receptor Gpr161 regulates forelimb formation, limb patterning and skeletal morphogenesis in a primary cilium-dependent manner. Development 2018. doi: 10.1242/dev.154054.

11. Tickle C. How the embryo makes a limb: determination, polarity and identity. J Anat 2015;227:418-30.

12. Zhang YT, Alber MS, Newman SA. Mathematical modeling of vertebrate limb development. Math Biosci 2013;243:1-17.

13. Schweitzer R, Chyung JH, Murtaugh LC, et al. Analysis of the tendon cell fate using Scleraxis, a specific marker for tendons and ligaments. Development 2001;128:3855-66.

14. Kardon G. Muscle and tendon morphogenesis in the avian hind limb. Development 1998;125:4019-32.

15. Guéro $S$. Developmental biology of the upper limb. Hand Surg Rehabil 2018;37:265-74.

16. Arvind V, Huang AH. Mechanobiology of limb musculoskeletal development. Ann N Y Acad Sci 2017;1409:18-32.

17. Di Giancamillo A, Deponti D, Raimondi MT, et al. Comparison between different cell sources and culture strategies for tendon tissue engineering. J Biol Regul Homeost Agents 2017;31:61-6.

18. Mazzocca AD, Chowaniec D, McCarthy MB, et al. In vitro changes in human tenocyte cultures obtained from proximal biceps tendon: multiple passages result in changes in routine cell markers. Knee Surg Sports Traumatol Arthrosc 2012;20:1666-72.

19. Ni M, Lui PP, Rui YF, et al. Tendon-derived stem cells (TDSCs) promote tendon repair in a rat patellar tendon window defect model. J Orthop Res 2012;30:613-9.

20. Costa-Almeida R, Calejo I, Gomes ME. Mesenchymal Stem Cells Empowering Tendon Regenerative Therapies. 
Int J Mol Sci 2019. doi: 10.3390/ijms20123002.

21. Takahashi K, Tanabe K, Ohnuki M, et al. Induction of pluripotent stem cells from adult human fibroblasts by defined factors. Cell 2007;131:861-72.

22. Gonzalez MA, Bernad A. Characteristics of Adult Stem Cells. Adv Exp Med Biol 2012;741:103-20.

23. Vieira CP, Viola M, Carneiro GD, et al. Glycine improves the remodeling process of tenocytes in vitro. Cell Biol Int 2018;42:804-14.

24. Cao Y, Liu YT, Liu W, et al. Bridging tendon defects using autologous tenocyte engineered tendon in a hen model. Plast Reconstr Surg 2002;110:1280-9.

25. Aparecida de Aro A, Vidal Bde C, Pimentel ER. Biochemical and anisotropical properties of tendons. Micron 2012;43:205-14.

26. Theiss F, Mirsaidi A, Mhanna R, et al. Use of biomimetic microtissue spheroids and specific growth factor supplementation to improve tenocyte differentiation and adaptation to a collagen-based scaffold in vitro. Biomaterials 2015;69:99-109.

27. di Giacomo V, Berardocco M, Gallorini M, et al. Combined supplementation of ascorbic acid and thyroid hormone $\mathrm{T} 3$ affects tenocyte proliferation. The effect of ascorbic acid in the production of nitric oxide. Muscles Ligaments Tendons J 2017;7:11-8.

28. Disser NP, Sugg KB, Talarek JR, et al. Insulin-like growth factor 1 signaling in tenocytes is required for adult tendon growth. FASEB J 2019;33:12680-95.

29. Alzyoud JAM, Al Najjar SA, Talat S, et al. Effect of light-emitting diodes, platelet-rich plasma, and their combination on the activity of sheep tenocytes. Lasers Med Sci 2019;34:759-66.

30. Wu YF, Huang YT, Wang HK, et al. Hyperglycemia Augments the Adipogenic Transdifferentiation Potential of Tenocytes and Is Alleviated by Cyclic Mechanical Stretch. Int J Mol Sci 2017. doi: 10.3390/ijms19010090.

31. Liu W, Chen B, Deng D, et al. Repair of tendon defect with dermal fibroblast engineered tendon in a porcine model. Tissue Eng 2006;12:775-88.

32. Deng D, Liu W, Xu F, et al. Engineering human neotendon tissue in vitro with human dermal fibroblasts under static mechanical strain. Biomaterials 2009;30:6724-30.

33. Wang W, He J, Feng B, et al. Aligned nanofibers direct human dermal fibroblasts to tenogenic phenotype in vitro and enhance tendon regeneration in vivo. Nanomedicine (Lond) 2016;11:1055-72.

34. Busra FM, Lokanathan Y, Nadzir MM, et al. Attachment, Proliferation, and Morphological Properties of Human
Dermal Fibroblasts on Ovine Tendon Collagen Scaffolds: A Comparative Study. Malays J Med Sci 2017;24:33-43.

35. Chen CH, Chen SH, Kuo CY, et al. Response of Dermal Fibroblasts to Biochemical and Physical Cues in Aligned Polycaprolactone/Silk Fibroin Nanofiber Scaffolds for Application in Tendon Tissue Engineering. Nanomaterials (Basel) 2017. doi: 10.3390/nano7080219.

36. Kwon J, Kim YH, Rhee SM, et al. Effects of Allogenic Dermal Fibroblasts on Rotator Cuff Healing in a Rabbit Model of Chronic Tear. Am J Sports Med 2018;46:1901-8.

37. Sensini A, Cristofolini L, Zucchelli A, et al. Hierarchical electrospun tendon-ligament bioinspired scaffolds induce changes in fibroblasts morphology under static and dynamic conditions. J Microsc 2019. [Epub ahead of print].

38. Nakanishi Y, Okada T, Takeuchi N, et al. Histological evaluation of tendon formation using a scaffold-free three-dimensional-bioprinted construct of human dermal fibroblasts under in vitro static tensile culture. Regen Ther 2019; $11: 47-55$.

39. Vapniarsky N, Arzi B, Hu JC, et al. Concise Review: Human Dermis as an Autologous Source of Stem Cells for Tissue Engineering and Regenerative Medicine. Stem Cells Translational Medicine 2015;4:1187-98.

40. Chen B, Ding J, Zhang W, et al. Tissue Engineering of Tendons: A Comparison of Muscle-Derived Cells, Tenocytes, and Dermal Fibroblasts as Cell Sources. Plast Reconstr Surg 2016;137:536e-44e.

41. Qiu Y, Wang X, Zhang Y, et al. In vitro two-dimensional and three-dimensional tenocyte culture for tendon tissue engineering. J Tissue Eng Regen Med 2016;10:E216-26.

42. Thulabandu V, Chen DM, Atit RP. Dermal fibroblast in cutaneous development and healing. Wiley Interdiscip Rev Dev Biol 2018. doi: 10.1002/wdev.307.

43. Dulak J, Szade K, Szade A, et al. Adult stem cells: hopes and hypes of regenerative medicine. Acta Biochim Pol 2015;62:329-37.

44. Yin Z, Chen X, Chen JL, et al. Stem cells for tendon tissue engineering and regeneration. Expert Opin Biol Ther 2010;10:689-700.

45. Li HL, Wei JF, Fan LY, et al. miR-302 regulates pluripotency, teratoma formation and differentiation in stem cells via an AKT1/OCT4-dependent manner. Cell Death Dis 2016;7:e2078.

46. Zheng YL. Some Ethical Concerns About Human Induced Pluripotent Stem Cells. Science and Engineering Ethics 2016;22:1277-84.

47. Harding J, Mirochnitchenko O. Preclinical studies for 
induced pluripotent stem cell-based therapeutics. J Biol Chem 2014;289:4585-93.

48. Dhinsa BS, Mahapatra AN, Khan WS. Sources of Adult Mesenchymal Stem Cells for Ligament and Tendon Tissue Engineering. Curr Stem Cell Res Ther 2015;10:26-30.

49. Lui PP. Stem cell technology for tendon regeneration: current status, challenges, and future research directions. Stem Cells Cloning 2015;8:163-74.

50. Font Tellado S, Balmayor ER, Van Griensven M. Strategies to engineer tendon/ligament-to-bone interface: Biomaterials, cells and growth factors. Adv Drug Deliv Rev 2015;94:126-40.

51. Bonab MM, Alimoghaddam K, Talebian F, et al. Aging of mesenchymal stem cell in vitro. BMC Cell Biol 2006;7:14.

52. Liu M, Lei H, Dong P, et al. Adipose-Derived Mesenchymal Stem Cells from the Elderly Exhibit Decreased Migration and Differentiation Abilities with Senescent Properties. Cell Transplantation 2017;26:1505-19.

53. Thomson JA, Itskovitz-Eldor J, Shapiro SS, et al. Embryonic stem cell lines derived from human blastocysts. Science 1998;282:1145-7.

54. Guillot PV, Cui W, Fisk NM, et al. Stem cell differentiation and expansion for clinical applications of tissue engineering. J Cell Mol Med 2007;11:935-44.

55. Chen X, Song XH, Yin Z, et al. Stepwise differentiation of human embryonic stem cells promotes tendon regeneration by secreting fetal tendon matrix and differentiation factors. Stem Cells 2009;27:1276-87.

56. Chen JL, Yin Z, Shen WL, et al. Efficacy of hESCMSCs in knitted silk-collagen scaffold for tendon tissue engineering and their roles. Biomaterials 2010;31:9438-51.

57. Schiele NR, Marturano JE, Kuo CK. Mechanical factors in embryonic tendon development: potential cues for stem cell tenogenesis. Curr Opin Biotechnol 2013;24:834-40.

58. Chen X, Yin Z, Chen JL, et al. Force and scleraxis synergistically promote the commitment of human ES cells derived MSCs to tenocytes. Sci Rep 2012;2:977.

59. Okech W, Kuo CK. Informing Stem Cell-Based Tendon Tissue Engineering Approaches with Embryonic Tendon Development. Adv Exp Med Biol 2016;920:63-77.

60. Dale TP, Mazher S, Webb WR, et al. Tenogenic Differentiation of Human Embryonic Stem Cells. Tissue Eng Part A 2018;24:361-8.

61. Takahashi K, Yamanaka S. Induction of pluripotent stem cells from mouse embryonic and adult fibroblast cultures by defined factors. Cell 2006;126:663-76.

62. Nethercott HE, Brick DJ, Schwartz PH. Derivation of induced pluripotent stem cells by lentiviral transduction. Methods Mol Biol 2011;767:67-85.

63. Lister R, Pelizzola M, Kida YS, et al. Hotspots of aberrant epigenomic reprogramming in human induced pluripotent stem cells. Nature 2011;471:68-73.

64. Engel M, Balez R, Munoz SS, et al. Viral-free generation and characterization of a human induced pluripotent stem cell line from dermal fibroblasts. Stem Cell Res 2018;32:135-8.

65. McGrath PS, Diette N, Kogut I, et al. RNA-based Reprogramming of Human Primary Fibroblasts into Induced Pluripotent Stem Cells. J Vis Exp 2018. doi: $10.3791 / 58687$.

66. Pittenger MF, Mackay AM, Beck SC, et al. Multilineage potential of adult human mesenchymal stem cells. Science 1999;284:143-7.

67. Samsonraj RM, Raghunath M, Nurcombe V, et al. Concise Review: Multifaceted Characterization of Human Mesenchymal Stem Cells for Use in Regenerative Medicine. Stem Cells Transl Med 2017;6:2173-85.

68. Rajpar I, Barrett JG. Optimizing growth factor induction of tenogenesis in three-dimensional culture of mesenchymal stem cells. J Tissue Eng 2019;10:2041731419848776.

69. Friedenstein AJ, Chailakhjan RK, Lalykina KS. The development of fibroblast colonies in monolayer cultures of guinea-pig bone marrow and spleen cells. Cell Tissue Kinet 1970;3:393-403.

70. da Silva Meirelles L, Chagastelles PC, Nardi NB. Mesenchymal stem cells reside in virtually all post-natal organs and tissues. J Cell Sci 2006;119:2204-13.

71. Lu W, Xu J, Dong S, et al. Anterior Cruciate Ligament Reconstruction in a Rabbit Model Using a Decellularized Allogenic Semitendinous Tendon Combined with Autologous Bone Marrow-Derived Mesenchymal Stem Cells. Stem Cells Transl Med 2019;8:971-82.

72. Awad HA, Boivin GP, Dressler MR, et al. Repair of patellar tendon injuries using a cell-collagen composite. Journal of Orthopaedic Research 2003;21:420-31.

73. Harris MT, Butler DL, Boivin GP, et al. Mesenchymal stem cells used for rabbit tendon repair can form ectopic bone and express alkaline phosphatase activity in constructs. J Orthop Res 2004;22:998-1003

74. Tan SL, Ahmad RE, Ahmad TS, et al. Effect of growth differentiation factor 5 on the proliferation and tenogenic differentiation potential of human mesenchymal stem cells in vitro. Cells Tissues Organs 2012;196:325-38.

75. Yin Z, Guo J, Wu TY, et al. Stepwise Differentiation of Mesenchymal Stem Cells Augments Tendon-Like Tissue 
Formation and Defect Repair In Vivo. Stem Cells Transl Med 2016;5:1106-16.

76. Bottagisio M, Lopa S, Granata V, et al. Different combinations of growth factors for the tenogenic differentiation of bone marrow mesenchymal stem cells in monolayer culture and in fibrin-based three-dimensional constructs. Differentiation 2017;95:44-53.

77. Le W, Yao J. The Effect of Myostatin (GDF-8) on Proliferation and Tenocyte Differentiation of Rat Bone Marrow-Derived Mesenchymal Stem Cells. J Hand Surg Asian Pac Vol 2017;22:200-7.

78. Zhang B, Luo Q, Deng B, et al. Construction of tendon replacement tissue based on collagen sponge and mesenchymal stem cells by coupled mechano-chemical induction and evaluation of its tendon repair abilities. Acta Biomater 2018;74:247-59.

79. Nam HY, Pingguan-Murphy B, Abbas AA, et al. Uniaxial Cyclic Tensile Stretching at 8\% Strain Exclusively Promotes Tenogenic Differentiation of Human Bone Marrow-Derived Mesenchymal Stromal Cells. Stem Cells Int 2019;2019:9723025.

80. Wang D, Jiang X, Lu A, et al. BMP14 induces tenogenic differentiation of bone marrow mesenchymal stem cells in vitro. Exp Ther Med 2018;16:1165-74.

81. Xie S, Zhou Y, Tang Y, et al. -Book-shaped decellularized tendon matrix scaffold combined with bone marrow mesenchymal stem cells-sheets for repair of achilles tendon defect in rabbit. J Orthop Res 2019;37:887-97.

82. Kryger GS, Chong AK, Costa M, et al. A comparison of tenocytes and mesenchymal stem cells for use in flexor tendon tissue engineering. J Hand Surg Am 2007;32:597-605.

83. Uysal AC, Mizuno H. Tendon regeneration and repair with adipose derived stem cells. Curr Stem Cell Res Ther 2010;5:161-7.

84. Almeida H, Domingues RMA, Mithieux SM, et al. Tropoelastin-Coated Tendon Biomimetic Scaffolds Promote Stem Cell Tenogenic Commitment and Deposition of Elastin-Rich Matrix. ACS Appl Mater Interfaces 2019;11:19830-40.

85. de Lima Santos A, Silva CGD, de Sa Barretto LS, et al. Biomechanical evaluation of tendon regeneration with adipose-derived stem cell. J Orthop Res 2019;37:1281-6.

86. Baer PC, Geiger H. Adipose-derived mesenchymal stromal/stem cells: tissue localization, characterization, and heterogeneity. Stem Cells Int 2012;2012:812693.

87. Yu Y, Zhou Y, Cheng T, et al. Hypoxia enhances tenocyte differentiation of adipose-derived mesenchymal stem cells by inducing hypoxia-inducible factor-1alpha in a coculture system. Cell Prolif 2016;49:173-84.

88. Farnebo S, Farnebo L, Kim M, et al. Optimized Repopulation of Tendon Hydrogel: Synergistic Effects of Growth Factor Combinations and Adipose-Derived Stem Cells. Hand (N Y) 2017;12:68-77.

89. Shen H, Jayaram R, Yoneda S, et al. The effect of adiposederived stem cell sheets and CTGF on early flexor tendon healing in a canine model. Sci Rep 2018;8:11078.

90. Chen QJ, Chen L, Wu SK, et al. rhPDGF-BB combined with ADSCs in the treatment of Achilles tendinitis via miR-363/PI3 K/Akt pathway. Mol Cell Biochem 2018;438:175-82.

91. Yang G, Rothrauff BB, Lin H, et al. Tendon-Derived Extracellular Matrix Enhances Transforming Growth Factor-beta3-Induced Tenogenic Differentiation of Human Adipose-Derived Stem Cells. Tissue Eng Part A 2017;23:166-76.

92. Gonçalves AI, Gershovich PM, Rodrigues MT, et al. Human adipose tissue-derived tenomodulin positive subpopulation of stem cells: A promising source of tendon progenitor cells. J Tissue Eng Regen Med 2018;12:762-74.

93. Subramanian G, Stasuk A, Elsaadany M, et al. Effect of Uniaxial Tensile Cyclic Loading Regimes on Matrix Organization and Tenogenic Differentiation of AdiposeDerived Stem Cells Encapsulated within 3D Collagen Scaffolds. Stem Cells Int 2017;2017:6072406.

94. Rinella L, Marano F, Paletto L, et al. Extracorporeal shock waves trigger tenogenic differentiation of human adiposederived stem cells. Connect Tissue Res 2018;59:561-73.

95. Bi Y, Ehirchiou D, Kilts TM, et al. Identification of tendon stem/progenitor cells and the role of the extracellular matrix in their niche. Nature Medicine 2007;13:1219-27.

96. Tan Q, Lui PP, Rui YF. Effect of in vitro passaging on the stem cell-related properties of tendon-derived stem cells-implications in tissue engineering. Stem Cells Dev 2012;21:790-800.

97. Jiang D, Xu B, Yang M, et al. Efficacy of tendon stem cells in fibroblast-derived matrix for tendon tissue engineering. Cytotherapy 2014;16:662-73.

98. Zhang YJ, Qing Q, Zhang YJ, et al. Enhancement of tenogenic differentiation of rat tendon-derived stem cells by biglycan. J Cell Physiol 2019. [Epub ahead of print].

99. Han P, Cui Q, Lu W, et al. Hepatocyte growth factor plays a dual role in tendon-derived stem cell proliferation, migration, and differentiation. J Cell Physiol 2019;234:17382-91.

100. Wang T, Thien C, Wang C, et al. 3D uniaxial mechanical 
stimulation induces tenogenic differentiation of tendonderived stem cells through a PI3K/AKT signaling pathway. FASEB J 2018;32:4804-14.

101. Lui PP, Wong OT, Lee YW. Transplantation of tendonderived stem cells pre-treated with connective tissue growth factor and ascorbic acid in vitro promoted better tendon repair in a patellar tendon window injury rat model. Cytotherapy 2016;18:99-112.

102.Zhang C, Zhang E, Yang L, et al. Histone deacetylase inhibitor treated cell sheet from mouse tendon stem/ progenitor cells promotes tendon repair. Biomaterials 2018;172:66-82.

103. Yin Z, Hu JJ, Yang L, et al. Single-cell analysis reveals a nestin+ tendon stem/progenitor cell population with strong tenogenic potentiality. Sci Adv 2016;2:e1600874.

104. Moshaverinia A, Xu X, Chen C, et al. Application of stem cells derived from the periodontal ligament or gingival tissue sources for tendon tissue regeneration. Biomaterials 2014;35:2642-50.

105. Chen YY, He ST, Yan FH, et al. Dental pulp stem cells express tendon markers under mechanical loading and are a potential cell source for tissue engineering of tendon-like tissue. Int J Oral Sci 2016;8:213-22.

106. Meirelles LS, Fontes AM, Covas DT, et al. Mechanisms involved in the therapeutic properties of mesenchymal stem cells. Cytokine Growth Factor Rev 2009;20:419-27.

107.Hsieh CF, Alberton P, Loffredo-Verde E, et al. Scaffoldfree Scleraxis-programmed tendon progenitors aid in significantly enhanced repair of full-size Achilles tendon rupture. Nanomedicine 2016;11:1153-67.

108.Behfar M, Javanmardi S, Sarrafzadeh-Rezaei

Cite this article as: Qi F, Deng Z, Ma Y, Wang S, Liu C, Lyu F, Wang T, Zheng Q. From the perspective of embryonic tendon development: various cells applied to tendon tissue engineering. Ann Transl Med 2020;8(4):131. doi: 10.21037/atm.2019.12.78
F. Comparative Study on Functional Effects of Allotransplantation of Bone Marrow Stromal Cells and Adipose Derived Stromal Vascular Fraction on Tendon Repair: A Biomechanical Study in Rabbits. Cell J 2014;16:263-70.

109. Mizuno H. Adipose-derived stem cells for tissue repair and regeneration: ten years of research and a literature review. J Nippon Med Sch 2009;76:56-66.

110.Hsiao ST, Asgari A, Lokmic Z, et al. Comparative analysis of paracrine factor expression in human adult mesenchymal stem cells derived from bone marrow, adipose, and dermal tissue. Stem Cells Dev 2012;21:2189-203.

111. Neo PY, See EYS, Toh SL, et al. Temporal profiling of the growth and multi-lineage potentiality of adipose tissuederived mesenchymal stem cells cell-sheets. J Tissue Eng Regen Med 2016;10:564-79.

112. Ni M, Rui YF, Tan Q, et al. Engineered scaffold-free tendon tissue produced by tendon-derived stem cells. Biomaterials 2013;34:2024-37.

113. Youngstrom DW, LaDow JE, Barrett JG. Tenogenesis of bone marrow-, adipose-, and tendon-derived stem cells in a dynamic bioreactor. Connect Tissue Res 2016;57:454-65.

114. Yan Z, Yin H, Nerlich M, et al. Boosting tendon repair: interplay of cells, growth factors and scaffold-free and gelbased carriers. J Exp Orthop 2018;5:1.

115.Zhang X, Lin YC, Rui YF, et al. Therapeutic Roles of Tendon Stem/Progenitor Cells in Tendinopathy. Stem Cells Int 2016;2016:4076578.

116.Liu C, Luo JW, Zhang KK, et al. Tendon-Derived Stem Cell Differentiation in the Degenerative Tendon Microenvironment. Stem Cells Int 2018;2018:2613821. 